\title{
Tratamiento del acretismo placentario: experiencia de un hospital público de Buenos Aires
}

\author{
Treatment of placenta accreta: experience of an University Public Hospital in \\ Buenos Aires
}

Gabriel Dionisio, Leandro Puerta, Natacha Ruiz, Sergio Brandeburgo, Carolina Etcheverry, Pablo Olmedo, Rubén Luca, María Celeste Simancas, Rubén Kevorkian, Sergio Centeno

\begin{abstract}
RESUMEN
Objetivos. El acretismo placentario (AP) constituye una situación de alta mortalidad maternofetal. Es el objetivo de este estudio describir la experiencia de nuestro centro en el tratamiento del AP y su influencia en la evolución natural de la patología. Material y métodos. Estudio clínico retrospectivo, unicéntrico, observacional. La estrategia terapéutica implementada fue embolización de arterias uterinas (EAU) seguida de histerectomía (EAUH) en la mayoría de los casos. Las variables continuas se expresaron como mediana y su respectivo intervalo intercuartilo 25-75, según su distribución, y las cualitativas en porcentajes.

Resultados. Fueron incluidas 44 pacientes, con una mediana de edad de 35 años (2244), en un período de 5 años, con diagnóstico de placenta previa o alto grado de sospecha de AP. El cateterismo de las arterias uterinas fue bilateral en un $90 \%(n=40)$, con técnica de cross-over. La EAU se realizó en un $81,8 \%$ de los casos $(n=35)$ con histerectomía posterior en un $86 \%(n=30)$. El 94,4\% fueron embolizadas en forma bilateral, con un resultado satisfactorio en el $97 \%(n=34)$ e insatisfactorio en uno de ellos. Detectamos un $22,7 \%$ de sangrado con requerimiento de transfusión $(n=10)$. Una paciente presentó embolización de sitio no deseado, con necrosis glútea y muerte $(2,85 \%)$

Conclusión: La implementación de una estrategia de EAU en pacientes portadoras de acretismo placentario, en un hospital público de la Ciudad de Buenos Aires, resulta compleja, pero factible. La mortalidad de las pacientes afectadas resultó casi tres veces menor que la reportada.
\end{abstract}

\begin{abstract}
Objective. Placenta accreta represents a situation of maternal and fetal high mortality rate. It's the aim of this study to describe the experience of our center in treatment of placenta accreta (PA).

Methods. Retrospective, single-center, observational clinical study. The therapeutic strategy was uterine artery embolization (UAE) followed by hysterectomy (UAEH) in most cases. Continuous variables were expressed as median and interquartile range 25 to 75 , according to their distribution, and qualitatives in percentages.

Results. 44 patients were included, with a median age of 35 years (22-44), over a period of five years, diagnosed with placenta previa or highly suspected placenta accreta. The uterine artery catheterization was performed bilaterally in $90 \%(n=40)$, with cross over technique. UAE was performed in $81.8 \%$ of cases $(n=35)$ with subsequent hysterectomy in $86 \%(n=30) .94 .4 \%$ were embolized bilaterally, with satisfactory results in $97 \%(n=34)$ and unsatisfactory in one. We detect $22.7 \%$ of bleeding with transfusion requirement $(n=10)$. In one patient non - wanted area embolization was detected, with gluteal necrosis and death (2.85\%)

Conclusion. The implementation of a strategy of UAE in patients with PA in a public hospital is complex, but doable. The mortality of patients affected was nearly three times lower than reported.
\end{abstract}

Key words: uterine artery embolization, postpartum hemorrhage, pregnancy.

Palabras claves: embolización de arterias uterinas, hemorragia posparto, embarazo.

Revista Argentina de Cardioangiología Intervencionista 2016;7(3):136-140

\section{INTRODUCCIÓN}

El acretismo placentario constituye una situación de alta mortalidad maternofetal. La hemorragia posparto que se produce como consecuencia de la placentación anormal es la principal causa de mortalidad materna en los países industrializados, y la tercera causa de morbilidad ligada al embarazo en todo el mundo ${ }^{1,2}$. Como consecuencia de la generalización del uso de la

1. Servicio de Hemodinamia. Hospital General de Agudos "DF Santojanni". CABA.

$\triangle$ Correspondencia: Gabriel Dionisio | Pilar 950, 1408 CABA, Rep. Argentina |Tel. (54011)5949-3309|gfdionisio75@yahoo.com.ar operación cesárea su incidencia ha aumentado 10 veces en los últimos 50 años, convirtiéndose en una patología de mayor frecuencia que la esperada, con una mortalidad de hasta un $7 \%^{2-4}$.

Esta enfermedad representa un desafío para el cirujano y un riesgo importante para la paciente. La intervención quirúrgica es compleja y conduce en la mayoría de los casos a la hemorragia masiva. Por otro lado, pueden afectarse estructuras vecinas, como el uréter y la vejiga, perjudicando aún más el pronóstico de la madre $e^{2-5}$. El tratamiento del acretismo placentario mediante la embolización de las arterias uterinas (EAU) permite evitar en muchos casos la histerectomía o, en su defecto, reducir considerablemente el riesgo de sangrado. Han sido propuestos diversos abordajes. Los primeros reportes reflejaban una elección por la intervención percutánea sólo frente a la falla de la estrategia 
quirúrgica. Posteriormente pudo observarse una conducta conservadora donde se prefería la no remoción de la placenta a la espera de su expulsión espontánea. Otros realizaban la embolización seguida de histerectomía en todos los casos ${ }^{3,5-11}$.

Es el objetivo de este estudio describir la experiencia de un hospital público universitario de la Ciudad de Buenos Aires en el abordaje y la estrategia terapéutica establecida en aquellas pacientes portadoras de acretismo placentario, y su influencia en la evolución natural de la patología.

\section{MATERIAL Y MÉTODOS}

\section{Diseño}

Se trata de un estudio clínico retrospectivo, unicéntrico, observacional. La estrategia terapéutica implementada inicialmente fue embolización de arterias uterinas (EAU) con intención de preservar la fertilidad $(n=5)$. Posteriormente se realizó histerectomía en todos los casos, lo que denominamos tratamiento híbrido (tratamiento endovascular más tratamiento quirúrgico).

El programa se dividió en dos períodos: de implementación inicial (P1), durante los primeros 12 meses $(\mathrm{n}=10)$; y de consolidación del programa (P2), a partir del segundo año ( 48 meses, $n=34$ ).

Las variables continuas se expresaron como mediana y su respectivo intervalo intercuartilo (II) 25-75, según su distribución, y las cualitativas como porcentajes.

\section{RESULTADOS}

\section{Población}

Fueron incluidas 44 pacientes, con una mediana de edad de 35 años (22-44), en un período de 5 años, con diagnóstico de placenta previa o alto grado de sospecha de acretismo placentario, definido por placenta inserta en área segmentaria anterior en la ecografía ginecobstétrica, y antecedentes de cirugía con afectación de la submucosa o mucosa, o cesáreas previas ${ }^{12,13}$.

Se excluyeron aquellas portadoras de fibroma voluminoso o múltiple, imposibilidad anatómica para realizar un cateterismo selectivo de las arterias uterinas, alergia comprobada a medios de contraste yodados o al material utilizado para la embolización (Spongostán ${ }^{\circ}$ ), insuficiencia renal en diálisis, imposibilidad para firmar y/o comprender el consentimiento informado o el procedimiento y/o la negativa de la familia y/o de la paciente.

\section{Sala de Hemodinamia}

Se colocó una vía periférica de grueso calibre y una sonda vesical con bolsa colectora. Luego de realizada limpieza con yodopovidona y una adecuada preparación del campo quirúrgico, se aplicó anestesia local de la zona inguinal, y se realizó una punción arterial e implante transitorio de dos introductores 5 o 6 French, en forma bilateral. Se infundieron 1000 UI de hepa-
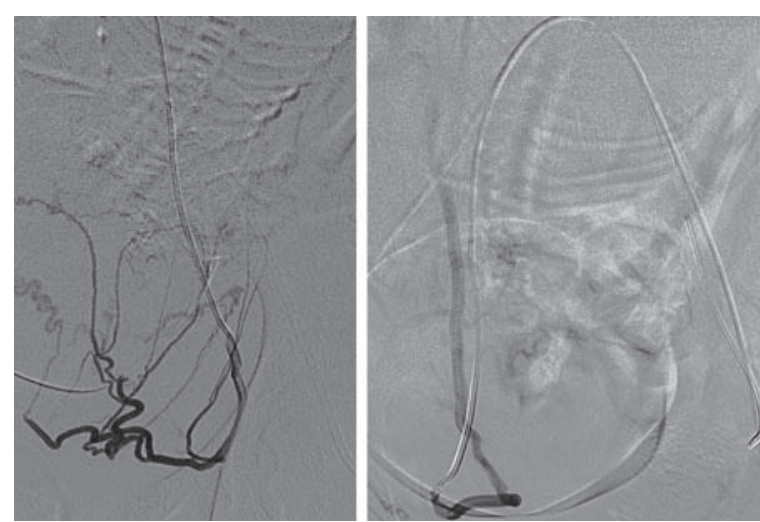

Figura 1. Arteriografía selectiva bilateral de arterias uterinas preembolización.

rina sódica diluidas en $5 \mathrm{ml}$ de solución fisiológica en bolo a través de la rama lateral de cada introductor arterial. Se progresó una cuerda hidrofílica 0,035" (Terumo") y se procedió al cateterismo selectivo contralateral de las arterias uterinas con dos catéteres 5 o 6 French tipo Cobra hidrofílicos (Terumo'). Se procedió a una angiografía selectiva para verificar la adecuada localización de los catéteres, la presencia de malformaciones vasculares y estimar la extensión de la invasión vascular trofoblástica (Figura 1). Se fijaron los introductores y catéteres con una sutura a piel por cada introductor y un film adhesivo estéril (Tegaderm)

\section{Quirófano}

Se recibió a la parturienta en el quirófano donde fue colocada en una camilla radiolúcida, con disponibilidad de 4 UI de glóbulos rojos, 4 UI de plasma y 6 de plaquetas, para la atención urgente de cualquier contingencia hemorrágica.

Luego de practicarle anestesia general, se prosiguió con el procedimiento obstétrico de laparotomía y operación cesárea con extracción del neonato. Luego de la extracción fetal se procedió a la administración de uterorretractores. Posteriormente, el hemodinamista realizó la EAU con Spongostán bajo control radiológico para comprobar el éxito de la embolización. El procedimiento se definió angiográficamente como satisfactorio, cuando se visualizaba stop circulatorio completo y persistente, insatisfactorio frente a la presencia de flujo en la arteria uterina y sus ramas, y regular al observarse lavado lento de contraste con flujo presente en arteria uterina principal.

Luego, se realizó histerectomía en la mayoría de los ca$\operatorname{sos}(86 \%, \mathrm{n}=30)$.

El control posoperatorio inmediato fue realizado en la Unidad de Terapia Intensiva. Luego de su estabilización y control evolutivo favorable, se procedió al pase a Sala General, con una mediana de 2 días de internación (II: 2-17).

La ecografía ginecológica fue suficiente para la toma de decisiones en la fase preoperatoria en la mayoría de los casos, siendo utilizada en el $86,4 \%$ de las pacientes. 
TABLA 1. Características basales de la población, procedimientos y resultados.

\begin{tabular}{|c|c|c|c|}
\hline \multicolumn{2}{|l|}{$\mathrm{N}$} & \multicolumn{2}{|l|}{44} \\
\hline \multicolumn{2}{|l|}{ Edad } & \multicolumn{2}{|l|}{35 (22-44) } \\
\hline \multicolumn{2}{|l|}{ Gestas } & \multicolumn{2}{|l|}{$5(1-10)$} \\
\hline \multicolumn{2}{|l|}{\begin{tabular}{|l|} 
Partos \\
\end{tabular}} & \multicolumn{2}{|l|}{$1(0-6)$} \\
\hline \multicolumn{2}{|l|}{ Abortos } & \multicolumn{2}{|l|}{$1(0-2)$} \\
\hline \multicolumn{2}{|l|}{ Cesáreas } & \multicolumn{2}{|l|}{$3(0-7)$} \\
\hline \multicolumn{2}{|l|}{ Semanas de gestación } & \multicolumn{2}{|l|}{$36(33-39)$} \\
\hline \multicolumn{2}{|l|}{ Embarazo gemelar } & \multicolumn{2}{|l|}{$2,2 \%$} \\
\hline \multicolumn{2}{|l|}{ Placenta previa } & \multicolumn{2}{|l|}{$79,6 \%$} \\
\hline \multicolumn{2}{|l|}{ Oclusiva } & \multicolumn{2}{|l|}{$85,7 \%$} \\
\hline \multicolumn{2}{|l|}{ Suboclusiva } & \multicolumn{2}{|l|}{$13,3 \%$} \\
\hline \multicolumn{2}{|l|}{\begin{tabular}{|l|} 
Anterior \\
\end{tabular}} & \multicolumn{2}{|l|}{$54,6 \%$} \\
\hline \multicolumn{2}{|l|}{ Posterior } & \multicolumn{2}{|l|}{$13,6 \%$} \\
\hline \multicolumn{2}{|l|}{ Compromiso órganos vecinos } & \multicolumn{2}{|l|}{$6,8 \%$} \\
\hline Eco ginecológica & $38(86,4 \%)$ & Cateterismo bilateral & 40 (90\%) \\
\hline RMN & $6(13,6 \%)$ & Embolización realizada & $35(82 \%)$ \\
\hline \multirow{3}{*}{ Protección radiante } & & Embolización no realizada & $9(18 \%)$ \\
\hline & $44(100 \%)$ & Sangrado mínimo y ausencia de acretismo & $8(89 \%)$ \\
\hline & & Pérdida de posición del catéter & $1(11 \%)$ \\
\hline Anestesia peridural & $3(6,8 \%)$ & Cateterismo ureteral & $2(4 \%)$ \\
\hline Anestesia general & $41(93,2 \%)$ & Días de internación & $2(2-17)$ \\
\hline Resultado angiográfico & & & \\
\hline Satisfactorio & & $34(97 \%)$ & \\
\hline Regular & & - & \\
\hline Insatisfactorio & & $1(3 \%)$ & \\
\hline Complicaciones vasculares & & $2,2 \% *$ & \\
\hline \begin{tabular}{|l|} 
Sangrado \\
Volumen de pérdida sanguínea
\end{tabular} & & $\begin{array}{l}22,7 \% \\
1500 \mathrm{ml}(500 \mathrm{ml}-2500 \mathrm{ml})\end{array}$ & \\
\hline Uso de hemoderivados*1 & & & \\
\hline Sangre & & $2(2-6)$ & \\
\hline Plasma & & $2(2-6)$ & \\
\hline Plaquetas & & $6(4-8)$ & \\
\hline Uso de coloides / cristaloides & & & \\
\hline Solución fisiológica & & $2500 \mathrm{ml}(1000 \mathrm{ml}-3500 \mathrm{ml})$ & \\
\hline Haemacell & & $1000 \mathrm{ml}(750 \mathrm{ml}-1000 \mathrm{ml})$ & \\
\hline Shock & & $2(4 \%)$ & \\
\hline Muerte P1; P2; Global & & 1 (10\%); 0 (0\%); 1 (2,85\%) & \\
\hline
\end{tabular}

* Representado por embolización de área no deseada $(n=1)$. * En pacientes con sanarado con reauerimiento de transfusión. P1: período 1. P2: período 2.

En seis casos el diagnóstico se realizó por medio de una RMN (13,6\%). En todas las pacientes se utilizó protección radiante. La anestesia general fue la más utilizada (93\%).

El cateterismo de las arterias uterinas se realizó en forma bilateral en un $90 \%$ de los casos $(n=40)$, con técnica de cross-over. Las cuatro pacientes restantes fueron cateterizadas en forma unilateral debido a la presencia de arteria uterina contralateral no significativa. El catéter de elección fue el Cobra 5 French en el $70 \%$ de los casos $(n=31)$, realizándose cateterismo ureteral en dos pacientes (4\%).

Fueron remitidas a Anatomía Patológica la placenta, o la placenta y el útero de las pacientes intervenidas en un $75 \%$ de los casos ( $n=33$ ). De este grupo, en un $61 \%$ se confirmó el diagnóstico de placentación anormal $(\mathrm{n}=20)$ predominantemente sin invasión más allá del endometrio $(75 \%, \mathrm{n}=15)$, con dos casos de percretismo (10\%) y tres de incretismo (15\%) (Figura 2).

La embolización se realizó en un $81,8 \%$ de los casos $(\mathrm{n}=35)$. La decisión de no realizarla surgió de la au- sencia de sangrado significativo y signos macroscópicos de acretismo placentario $(\mathrm{n}=8)$. En un caso no se realizó al comprobar en quirófano por radioscopia, antes de la extracción del útero, la pérdida de posición del catéter del sitio adecuado. El 94,4\% fueron embolizadas en forma bilateral. En todos los casos se utilizó Spongostán, con un resultado satisfactorio en un $97,15 \%(n=34)$ e insatisfactorio en uno de ellos $(2,85 \%)$. No detectamos complicaciones del sitio de punción. Detectamos un $22,7 \%$ de sangrado con requerimiento de transfusión $(\mathrm{n}=10)$, con una mediana de volumen de pérdida de sangre de $1500 \mathrm{ml}$ (5002500). Este grupo de pacientes recibió transfusión de glóbulos rojos deplasmatizados en todos los casos, con una mediana de 2 unidades internacionales (UI) (II: 2-6). Se administró plasma en el $70 \%$ de los pacientes con sangrado, con una mediana de 2 unidades (II: 2-6) El 80\% de estos pacientes requirieron expansión con cristaloides y coloides con una mediana de $2500 \mathrm{ml}$ de solución fisiológica (II: 1000-3500) y $1000 \mathrm{ml}$ de coloides (II: 750-1000). Dos pacientes 


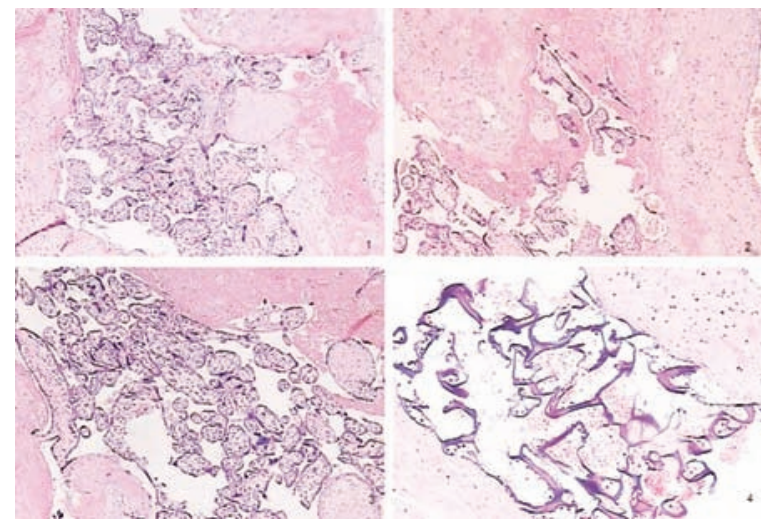

Figura 2. Histopatología de los preparados anatomopatológicos obtenidos. 1 y 2. Vellosidades coriales invadiendo superficialmente el miometrio (H-E 5X). 3. Vellosidades coriales invadiendo en profundidad el miometrio (H-E 5X). 4. Spongostán adherido al miometrio (H-E 10X). H-E 5X: hematoxilina y eosina a 5 aumentos. H-E 10X: hematoxilina y eosina a 10 aumentos.

evolucionaron con shock (4\%). Todos los recién nacidos presentaron vitalidad adecuada (APGAR $\geq 8$ ).

Durante el período de implementación inicial, una paciente en shock hipovolémico presentó embolización de sitio no deseado, necrosis glútea y del miembro inferior izquierdo, necesidad de amputación y muerte $\left(17^{\circ}\right.$ día de internación). La mortalidad durante el P1 fue de un $10 \%$, en el P2 fue $0 \%$ y la mortalidad global fue 2,85\% (Tabla 1, Gráfico 1).

\section{DISCUSIÓN}

La primera etapa del programa permitió el desarrollo de la técnica, interactuar con los diversos servicios involucrados y formar un grupo multidisciplinario de trabajo eficiente. Se detectaron las debilidades del mismo y fueron corregidas. Estas derivaban de la dificultad de establecer una coordinación adecuada entre los cirujanos obstetras y el grupo de hemodinamia $(\mathrm{GH})$, la preparación de un quirófano que respondiera a las necesidades radiológicas y a los requerimientos de anestesia para la intubación orotraqueal y monitoreo, y la incorporación del GH, por parte del personal de quirófano, como un elemento propio. Otro desafío fue el entrenamiento de los profesionales de Terapia Intensiva sobre el control de las posibles complicaciones vinculadas al procedimiento y la necesidad de contar con un banco de sangre suficiente para resolver contingencias hemorrágicas.

El segundo período fue el de consolidación. Durante el mismo todo el GH pudo desarrollar la técnica, siendo más accesible para la Institución y los profesionales involucrados. Pudieron articularse actividades propendientes a mejorar los resultados, como la presentación de cada caso en ateneo multidisciplinario previo al procedimiento.

La población intervenida estuvo compuesta por un grupo de pacientes adultas jóvenes, multíparas, con antecedente de frecuentes operaciones cesárea. En la mayoría de los casos se detectó placenta previa $(80 \%)$ y

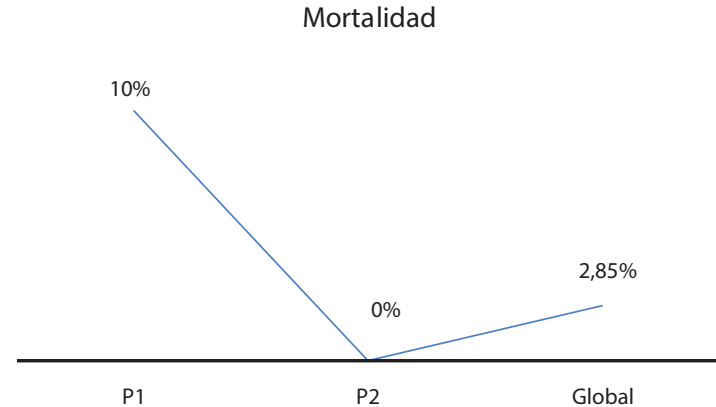

Gráfico 1. Diferencias de mortalidad entre el período 1 (P1) y 2 (P2), y mortalidad global.

de tipo oclusiva (85,7\%). La ubicación anterior se presentó en más de la mitad de los casos $(54,6 \%)$. La invasión trofoblástica extrauterina fue infrecuente $(6,8 \%)$. El estudio preoperatorio de elección para establecer el diagnóstico de acretismo placentario fue la ecografía ginecológica $(86,4 \%)$, relegando la resonancia magnética nuclear a pocos casos.

Se prestó particular atención a la protección radiológica del feto, independientemente de que en el período de gestación alcanzado era menos probable su afectación por radiación ionizante (mediana 36 semanas, II: 33-39).

La vía de canulación de elección fue la femoral contralateral. La ausencia de quirófano híbrido no resultó un impedimento para realizar los procedimientos; sin embargo, las limitaciones del arco en "C" impidieron la recanulación del vaso en un caso en que se desplazó el catéter.

El Servicio de Anestesiología optó, luego de la experiencia inicial, por la anestesia general sobre la peridural; fundamentalmente debido a la estrategia establecida de histerectomía posembolización. Por otro lado, la necesidad de traslado luego de la canulación desde el Servicio de Hemodinamia a quirófano, hacía complejo el uso de anestesia peridural.

La tasa de embolización, embolización bilateral y embolización satisfactoria fue elevada, según la información que ha podido obtenerse de los casos reportados ${ }^{14,15}$.

El sangrado significativo que requirió transfusión tuvo una tasa que coincide con la reportada, y pudo ser controlado. El volumen de sangrado detectado resultó inferior al reportado en la literatura ${ }^{16-18}$. La frecuencia de shock fue baja.

Debido a que la población de referencia corresponde a un grupo socioeconómico desfavorecido, sin acceso a educación reproductiva, alta tasa de maternidad precoz y madres multíparas, teniendo en cuenta el riesgo del procedimiento y obedeciendo al deseo de las pacientes y sus familiares, se optó por no preservar la fer- 
tilidad luego de los primeros casos. El hecho de haber establecido una estrategia de histerectomía posembolización puede explicar, al menos en parte, la embolización de área no deseada detectada en una paciente de la muestra. Publicaciones previas reportan que la manipulación enérgica del paquete vascular uterino puede representar una fuente de reflujo del material utilizado en una EAU. ${ }^{1}$

Debemos destacar que se produjo durante el período inicial (caso $\mathrm{n}^{\circ}$ 5).

La posibilidad de ofrecer una terapéutica de estas características a pacientes con difícil acceso al seguimiento terapéutico preparto, refleja el resultado del esfuerzo y la coordinación de diversos sectores de un grupo multidisciplinario. La división del programa en dos etapas permitió establecer objetivos acordes a cada período. De esta manera, los resultados iniciales no detuvieron el proceso hasta la consolidación del grupo.

La implementación de una estrategia híbrida de EAUH, en pacientes portadoras de acretismo placen-

\section{BIBLIOGRAFÍA}

1. Ojala K, PeräläJ, Kariniemi J, Ranta P, Raudaskoski T, Tekay A. Arterialembolization and prophylactic catheterization for the treatment for severe obstetric hemorrhage. Acta Obst Gynecol Scand 2005:84(11):1075-80.

2. Omar Dueñas G., Hugo Rico O.,Mario Rodríguez B. Actualidad en el diagnóstico y manejo del acretismo placentario. Rev Chil Obstet Ginecol 2007;72(4):266-74.

3. Angstmann T, Gard G, Harrington T, et al. Surgical management of placenta accreta: a cohort series and suggested approach. Am J Obstet Gynecol 2010;202:38.e1-9.

4. Kayem G, Davy C, Goffinet F, Thomas C, Clément D, Cabrol D. Conservative versus extirpative management in cases of placenta accreta. Obstet Gynecol 2004 Sep;104(3):531-6.

5. Chan BC, Lam HS, Yuen JH, Lam TP, Tso WK, Pun TC, Lee CP. Conservative management of placenta praevia with accreta. Hong Kong Med J. 2008 Dec;14(6):479-84.

6. Weinstein A, Chandra P, Schiavello H, Fleischer A. Conservative management of placenta previa percreta in a Jehovah's Witness. Obstet Gynecol. 2005 May; 105(5 Pt 2):1247-50

7. Wee L, Barron J, Toye R. Management of severe postpartum haemorrhage by uterine artery embolization. Br J Anaesth. 2004 Oct;93(4):5914. Epub $2004 \mathrm{Jul} 26$.

8. L.J.Salomon, R.deTayrac, V.Castaigne-Meary et al. Fertility and pregnancy outcome following pelvic arterial embolization for severe post-partum haemorrhage. Acohort study. Human Reproduction Vol.18, No.4 pp. 849 $\pm 852,2003$.

9. David Ornan, Robert White,MD, Jeffrey Pollak, MD, and Michael Tal, MD. Pelvic Embolization for Intractable PostpartumHemorrhage: Long-Term Follow-up and Implicationsfor Fertility. Obstet Gynecol 2003;102:904-10. tario en un hospital público universitario de la Ciudad de Buenos Aires, resulta compleja, pero factible.

En nuestra experiencia, la EAU seguida de histerectomía en pacientes cursando acretismo placentario redujo algo más de dos veces la mortalidad reportada. Superada la etapa inicial, los resultados en términos de morbimortalidad fueron óptimos.

\section{Limitaciones del estudio}

La estrategia implementada en la mayoría de los casos (EAU seguida de histerectomía) impide valorar el beneficio de preservar la capacidad reproductiva. Tampoco nos permite evaluar cómo habrían de modificarse los resultados en otros aspectos relacionados (síndrome posembolización, fiebre, infecciones, días de internación, etc.).

La afirmación sobre las diferencias en la mortalidad con respecto a los valores reportados en la literatura no pueden ser tomadas como definitorias en estudios observacionales.

10. Brown BJ et al. Uncontrolable post - partum bleeding: a new approach to hemostasis through angiographic arterial embolization. Obstet Gynecol 1979; 54: $361-5$

11. Harold A. Mitty MD, Keith M. Sterling MD, Manuel Alvarez MD et al. Obstetric Hemorrhage: Prophylacticand Emergency Arterial Catheterization and Embolotherapy. Radiology 1993; 188:183-189.

12. Oppenheimer L, Society of Obstetricians and Gynaecologists of Canada. Diagnosis and management of placenta previa. J Obstet Gynaecol Can 2007 Mar;29(3):261-6.[59 references]

13. Royal College of Obstetricians and Gynaecologists (RCOG). Placenta praevia and placenta praevia accreta: diagnosisand management. London (UK): Royal College of Obstetricians and Gynaecologists (RCOG);2005 Oct. 12p. (Guideline; no. 27). [97 references].

14. Descargues G, Douvrin F, LemoineJP, Marpeau L, Clavier E. Abnormal. placentation and selective embolization of the uterine arteries. Eur J ObstetGynecol Reprod Biol 2001; 99: 47-52.

15. Pelage JP, Le Dref O, Mateo J, Soyer P, Jacob D et al. Lifethreatening primary. postpartum hemorrhage: treatmentwith emergency selective arterial embolization Radiology 1998; 208: 359-62.

16. Wu S, Kocherginsky M, Hibbard JU. Abnormal placentation: twenty-year analysis. Am JObstet Gynecol 2005;192:1458-61.

17. Leung T-K, Au H-K, Lee C-M, et al. Prophylactic trans-uterine embolization to reduce intraoperative blood loss for placenta percreta invading the urinary bladder. J Obstet Gynaecol Res 2007;33:722-5.

18. ShrivastavaV, NageotteM, MajorC, HaydonM, Wing D. Case-controlcomparison of cesarean hysterectomy with and without prophylactic placement of intravascular balloon cathetersfor placenta accreta. Am J Obstet Gynecol2007;197:402.e 1-5. 